\title{
LES PHLÉBOTOMES DU PORTUGAL
}

I - Infestation naturelle

de Phlebotomus ariasi Tonnoir, 1921

et Phlebotomus perniciosus Newstead, 1911,

par Leishmania dans le foyer zoonotique

de Arrábida (Portugal) ${ }^{1}$

\author{
C. A. PIRES*
}

RÉSUMÉ. L'auteur présente les résultats d'une enquête sur les Phlébotomes dans le foyer zoonotique de Arrábida (Portugal) pour déterminer les vecteurs du kala-azar. Ont été identifiées trois espèces de Phlébotomes : Sergentomyia (S.) minuta, Phlebotomus (L.) ariasi et Phlebotomus (L.) perniciosus; seules les deux dernières espèces ont été trouvées parasitées par des promastigotes de Leishmania : 2 femelles de $P$. ariasi $(4,88 \%)$ et 3 femelles de $P$. perniciosus $(2,01 \%)$ sur un total de 195 femelles disséquées.

C'est aussi la première observation de l'infestation naturelle de $P$. ariasi au Portugal.

\section{The Phlebotomine sandflies of Portugal. I - Natural infection of Phlebotomus ariasi Tonnoir, 1921 and Phlebotomus perniciosus Newstead, 1911 by Leish- mania in zoonotic focus of Arrábida (Portugal)}

SUMMARY. The author presents the results of a sandfly survey carried out in the zoonotic focus of Arrábida for search of kala-azar vectors. The total of 195 dissected females belonged to the species Sergentomyia (S.) minuta. Phlebotomus (L.) ariasi and Phlebotomus (L.) perniciosus. Only the last two species were found parasitized by Leishmania promastigotes : 2 females of $P$. ariasi $(4.88 \%)$ and 3 females of $P$. perniciosus $(2.01 \%)$.

This is the first finding of natural infection of $P$. ariasi in Portugal.

Une récente enquête sur le kala-azar réalisée dans les environs de Lisbonne (P. Abranches et coll., 1983a et b) avait montré l'existence de la maladie canine (fréquence $11,6 \%$ ) et vulpine $(4,17 \%$ ) dans la région de Serra de Arrábida-S. Luis.

I. Ce travail a été subventionné partiellement par le "Centro das Doenças Infecciosas e Parasitarias da Universidade Nova de Lisboa".

* Instituto de Higiene e Medicina Tropical, 96 Rua da Junqueira, 1300 Lisboa, Portugal.

Accepté le 24 novembre 1983 . 
On connaissait déjà l'existence, dans le foyer de Arrábida de Phlebotomus perniciosus et $P$. ariasi (C. A. Pires, 1979) qui sont les vecteurs des leishmanioses dans la partie occidentale de la sous-région méditerranéenne (J. A. Rioux et coll., 1969, R. KillickKendrick, 1978).

Afin d'identifier le vecteur dans ce foyer nous avons effectué une série de captures dans cette même zone (juillet à septembre). Les captures ont été réalisées au moyen d'aspirateur électrique dans des abris naturels, des chenils, et des habitations. Les Phlébotomes transportés au laboratoire furent disséqués en solution saline (J. A. Rioux et coll., 1972). Le tube digestif, les ovaires et la tête ont été placés entre lame et lamelle. L'identification a été établie par l'examen des spermatèques.

Sur un total de 195 q disséquées, trois espèces ont pu être identifiées : Sergentomyia (S.) mimuta (Rondani, 1843), Phlebotomus (L.) ariasi Tonnoir, 1921 et Phlebotomus (L.) perniciosus Newstead, 1911.

Les parasites ont été observés dans 2 Phlébotomus ariasi sur 41 (4,88\%) et 3 Phlebotomus perniciosus sur $149(2,01 \%)\left(\mathrm{X}^{2}=1,030 ; \mathrm{P} \gg 0,05\right.$ non significatif) (tableau I).

Tableau I. - Fréquence du parasitisme chez les 195 Phlébotomes capturées.

\begin{tabular}{lccc}
\hline & $\begin{array}{c}\text { Nombre de }+ \\
\text { examinées }\end{array}$ & $\begin{array}{c}\text { Nombre de }+ \\
\text { hébergeant } \\
\text { les formes } \\
\text { promastigotes }\end{array}$ & $\begin{array}{c}\% \text { de } q \\
\text { infestées }\end{array}$ \\
\hline S. minuta (Rond.) & 5 & 0 & 0,0 \\
$P$. ariasi Tonnoir & 41 & 2 & 4,88 \\
$P$. perniciosus Newst. & 149 & 3 & 2,01 \\
$\quad$ Total & 195 & 5 & 2,56 \\
\hline
\end{tabular}

Les 2 exemplaires $q$ de $P$. ariasi parasités présentaient du sang dans l'intestin. L'un ( $\left.9 \mathrm{n}^{\circ} 76\right)$ abritait un grand nombre de promastigotes au niveau de la valve œsophagienne. Les ovaires étaient au stade III et IV. Les 3 femelles parasitées de $P$. perniciosus ne présentaient aucun vestige sanguin. Deux d'entre elles (q no 2 et $n^{\circ} 73$ ) contenaient des promastigotes dans l'intestin moyen et la valve œsophagienne. Les ovaires étaient au stade III chez deux exemplaires et au stade V chez le troisième. Sur les 5 femelles parasitées, 4 ( $2 P$. ariasi et $2 P$. perniciosus) avaient été capturées dans le même biotope de la région de Casais da Serra (fig. 1), à $300 \mathrm{~m}$ environ d'une habitation, la dernière provenait d'un chenil de Aldeia Grande. 


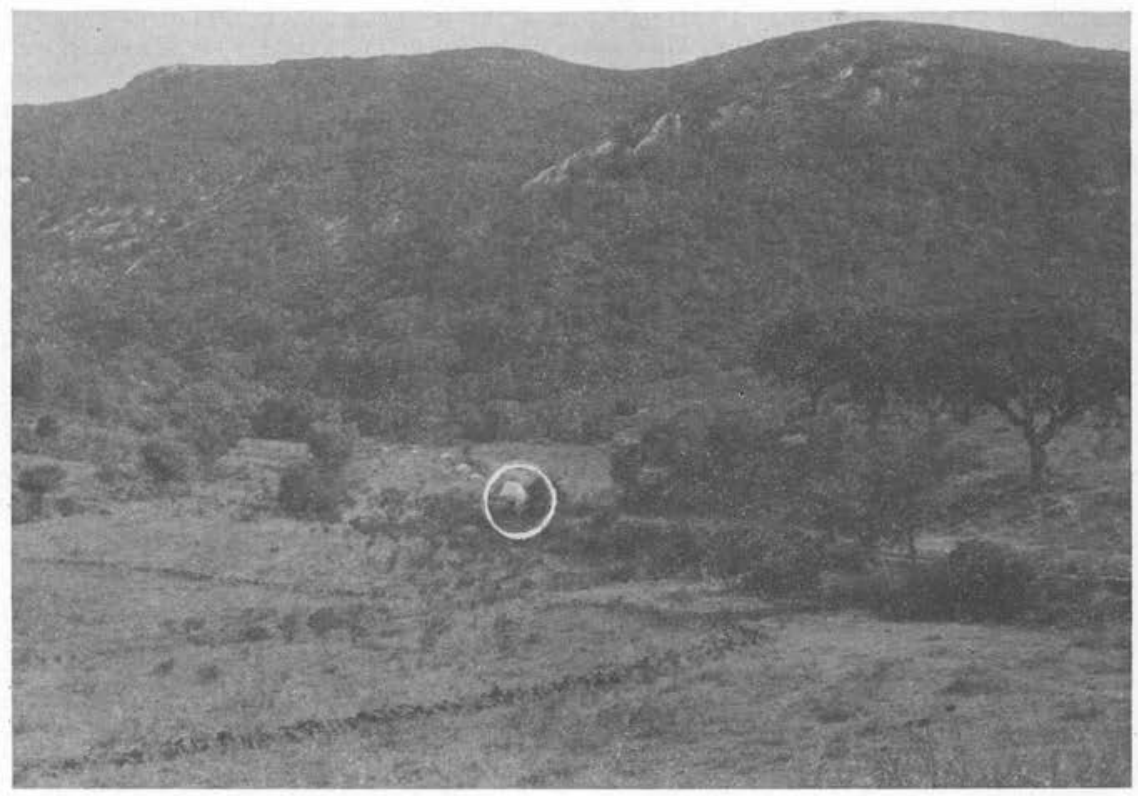

FIG. I. - Casais da Serra : Site de capture de Phlebotomus ariasi Tonn. et Phlebotomus perniciosus Newst. parasités. Alt. $135 \mathrm{~m}$. Au premier plan, pâturages et Oliviers épars. A l'arrière plan sur les pentes de Serra de Arrabida la suberaie (Quercus suber).

Les résultats obtenus montrent que les deux espèces peuvent être également parasitées dans le même foyer. Des études ultérieures sur l'écosystème de la zone d'Arrabida s'avèrent nécessaires pour déterminer le rôle vectoriel de chacune des espèces. Cependant on peut déjà signaler deux faits marquants :

- La première mention de l'infestation naturelle de $P$. ariasi au Portugal.

- L'infestation naturelle simultanée de $P$. ariasi et de $P$. perniciosus dans le même foyer endémique de Arrábida.

Il reste à présent à identifier par les techniques enzymatiques, les parasites observés et à déterminer le rôle de chacune des deux espèces de Phlébotome dans l'entretien du cycle domestique et, s'il est effectif, du cycle sauvage.

Remerciements. Nous remercions vivement MM. les Professeurs H. Ribeiro et J. A. Rioux ainsi que le Docteur P. Abranches qui nous ont fait profiter largement de leur expérience.

\section{BIBLTOGRAPHIE}

Abranches P., Lopes F. J., Silva F. M. C., Ribeiro M. M. S., Pires C. A. : - Le Kala-Azar au Portugal III. - Résultats d'une enquête sur la leishmaniose canine réalisée dans les environs de Lisbonne. Comparaison des zones urbaines et rurales. Ann. Parasitol. Hum. Comp., I983a, s8, 307-315. 
Abranches P., Conceiçao Sillya F. M., Ribeiro M. M. S., Lopes F. J., Teixeira Gomes L. : The Kala-azar in Portugal - IV. The isolation of a Leishmania from a fox. Trans. Roy. Soc. Trop. Med. Hyg., $\mathrm{I}_{9} 83 b, 73,420-421$.

Dolmatova A. V., Demina N. A. : Les Phlébotomes (Phlebotominae) et les maladies qu'ils transmettent. Initiations-Docum. Techn. ORSTOM, 1971, I8, 168 p.

Girao A. A. : Geografia de Portugal. VII. Clima e vegetaçao. Regioes Naturais. Portucalense Editora, I94I, 479 p.

KILLICK-KENDRICK R. : Recent advances and outstanding problems in the biology of Phlebotomine sandflies. A review. Acta Trop., 1978, 35, 297-313.

LAmotre M. : Introduction à la biologie quantitative. Masson, Paris, 1958 .

PIRes C. A. : Contribuiçao ao conhecimento da distribuiçao e bioecologia dos flebótomos de Portugal. Bolm Soc. port. Ciênc. nat., 1979, 19, 197-210.

Rioux J.-A., Golvan Y. J., Croset H., Tour S., Houin R., Abonnenc E., Petitdidier H. Vollahrdt Y., Dedet J. P., Albaret J. L., Lannote G., Quillici M. : Epidemiologie des leishmanioses dans le Sud de la France. Monographie INSERM, 1969, 37, 223 p.

Rioux J.-A., Lanotre G., Croset H., Deder J.-P. : Écologie des leishmanioses dans le Sud de la France. 5 - Pouvoir infestant comparé des diverses formes de leishmaniose canine vis-à-vis de Phlebotomus ariasi Tonnoir, 1921. Ann. Parasitol. Hum. Comp., 1978, 47, 413-419. 